\title{
Study on the Generation of Ultra-wideband Doublet Pulse Based on SOA-XPM in a Nonlinear Optical Loop Mirror
}

\author{
Yalin Guan, Zhen Zhang, Xinqiao Chen, Ruidong Wang \\ College of Information Engineering, Communication University of China, Beijing 100024, China \\ 234485920@163.com
}

\begin{abstract}
Photonic generation of UWB signals is the one of the key technology of UWB-over-fiber system. This paper proposes a method to generate UWB doublet signals utilizing the cross-phase modulation (XPM) in a semiconductor optical amplifier (SOA) in a NOLM. The simulation experiment based on the proposed method is carried out. UWB doublet signals which have a central frequency of $6.05 \mathrm{GHz}$ and a relative bandwidth of $121 \%$ are obtained in the simulation experiment. The simulation result verifies the feasibility of the proposed method. In addition, the central frequency and bandwidth of the generated UWB doublet signal is variable.

Index Terms - Ultra-wideband (UWB) signal, UWB doublet, UWB over fiber, nonlinear optical loop mirror (NOLM), semiconductor optical amplifier (SOA).
\end{abstract}

\section{Introduction}

Ultra-wideband (UWB) technology has attracted widespread attention in the field of wireless communication and sensor network in recent years because of its advantages, such as the immunity to multi-path fading, high data rate, low power consumption, and broad bandwidth. According to the U.S. Federal Communications Commission (FCC), a UWB signal should have a spectral bandwidth that is greater than $500 \mathrm{MHz}$ or a fractional bandwidth that is greater than $20 \%$. In indoor UWB systems, it should have a frequency bands between 3.1 to $10.6 \mathrm{GHz}$ with a power density lower than $-41.3 \mathrm{dBm} / \mathrm{MHz}$ [1-2]. Due to its low power spectral density, the communication distances of UWB system are limited. Thanks to the low loss and broad bandwidth of optical fiber, Ultra-wideband (UWB) signals over optical fiber (UWB over fiber) is proposed to extend the distances of UWB communication.

Generating UWB signal directly in the optical domain without the need for extra optical-electrical and electro-optical conversions is a key technology in UWB over fiber systems. It also has many other advantages, such as small size, large tenability, immunity to electromagnetic interference, and so on. On the other hand, through mathematical analysis we can know that UWB doublet has a better conformity to the spectrum regulated by FCC than UWB monocycle. So it has been widely studied in the UWB systems [3]. The basic principle has been proposed to generate UWB pulses in the optical domain can be classified into three categories: 1) UWB pulse generation based on phase-modulation-to-intensitymodulation (PM-IM) conversion. 2) UWB pulse generation using a photonic microwave delay-line filter. 3) UWB pulse generation based on optical spectral shaping and dispersioninduced frequency-to-time mapping [4-5].
Nonlinear optical loop mirror (NOLM) is proposed by Doran N.J. in 1988 [6]. Due to the development of all-optical network, NOLM has been widely studied in optical wavelength converter, optical logic gate, optical switches, and so on. This paper proposes a method to generate UWB doublet signals utilizing the cross-phase modulation (XPM) in a semiconductor optical amplifier (SOA) in a NOLM. The NOLM is used to implement the PM-IM conversion. And UWB doublet pulses are obtained from the simulation experiment based on the proposed method. The central frequency and $-10 \mathrm{~dB}$ bandwidth tenability of the generated UWB signal is also discussed.

\section{Operation Principle}

The scheme of UWB doublet pulse generation based on SOA-XPM in a NOLM is shown in Fig. 1. The input optical signal is split to two parts by an OC with a split ratio of 0.5 . These two parts are launched into the clockwise $(\mathrm{CW})$ and the counter-clockwise (CCW) of the SOA based NOLM, respectively. The optical field of these two parts can be written as

$$
\begin{aligned}
& E_{C W}(t)=\frac{\sqrt{2}}{2} E_{\text {in }}(t) \\
& E_{C C W}(t)=\frac{\sqrt{2}}{2} E_{\text {in }}(t) \exp \left(j \frac{\pi}{2}\right)
\end{aligned}
$$

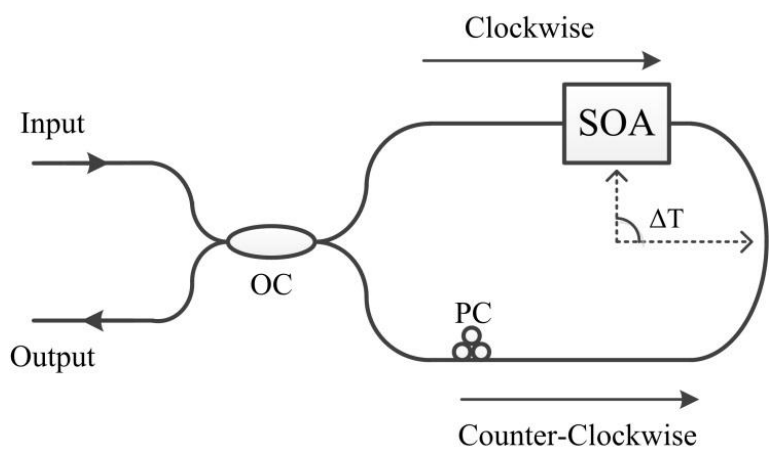

Fig. 1 The scheme of UWB doublet pulse generation based on SOA-XPM in a NOLM

where $E_{\text {in }}$ is the input optical field. Assume that the time delay between the SOA and the middle of the NOLM is $\Delta \mathrm{T}$, as shown in Fig. 1. So the CCW light will have a $2 \Delta \mathrm{T}$ time delay to enter the SOA comparing with the $\mathrm{CW}$ light. Due to the 
XPM effect in the SOA, the CW and CCW light are both phase modulate by each other. The phase shift $\phi$ is proportional with the power of the $\mathrm{CCW}$ and $\mathrm{CW}$ light, respectively. Thus their optical fields can be rewritten as

$$
\begin{gathered}
E_{C}(t)=\frac{\sqrt{2 G}}{2} E_{\text {in }}(t) \exp \left\{j\left[\phi\left(P_{C C W}\right)\right]\right\} \\
E_{C C}(t)=\frac{\sqrt{2 G}}{2} E_{\text {in }}(t) \exp \left\{j\left[\phi\left(P_{C W}\right)+\frac{\pi}{2}\right]\right\}
\end{gathered}
$$

where $P_{\text {CWW }}$ and $P_{\text {CCW }}$ are the intensity of the $\mathrm{CW}$ and $\mathrm{CCW}$ lights, respectively. $\phi$ is the phase shift introduced by the XPM effect of the SOA which is a function about $P_{C W}$ and $P_{\text {CCW }} . \mathrm{G}$ is the gain index of the SOA. Then the CW light and the CCW light are recombined and interference at the $\mathrm{OC}$ and the power of the output light can be written as

$$
P_{\text {out }}=G P_{\text {in }} \cos ^{2}\left[\frac{\phi\left[P_{\mathrm{CW}}\right)-\phi\left[P_{\mathrm{CCW}}{ }^{\jmath}\right.}{2}\right]
$$

where $P_{\text {in }}$ is the intensity of the input light. From Eq. (5), the phase shift of the $\mathrm{CW}$ and $\mathrm{CCW}$ lights are converted to the intensity of the input light. Assume that the input is a Gaussian pulse. According to the description above, the moment of the phase shift of the CW and CCW lights have $2 \Delta \mathrm{T}$ delay and $-2 \Delta \mathrm{T}$ ahead, comparing with the peak of the input Gaussian pulse, respectively. These two phase shift are converted to two intensity notches in the output light. These two notches are distributed in the two sides of the peak symmetrically, as shown in Fig. 2.

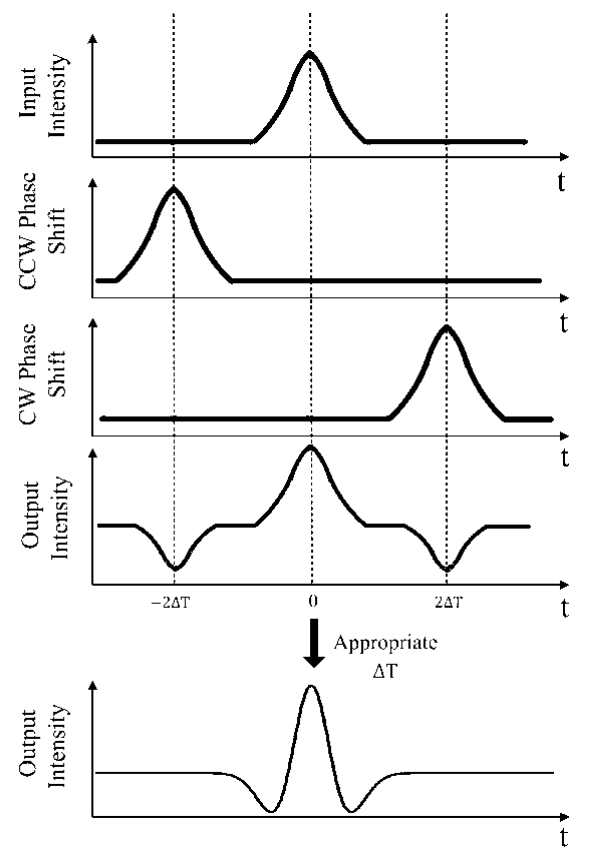

Fig. 2 The conversion from phase-modulation to intensity-modulation

By changing the time delay $\Delta \mathrm{T}$, these two notches can be closed to the peak. When $\Delta \mathrm{T}$ has an appropriate value, the output light shows a UWB doublet pulse shape.

\section{Simulation Experiment and Discussion}

The simulation experiment scheme based on the proposed method is shown in Fig. 3. Due to the unavailability of the bidirectional optical transmission in the simulation software, the NOLM is designed in such a way that the CW and CCW light will transmission separately [7]. The input light which modulated by Gaussian pulse is separated to the $\mathrm{CW}$ and $\mathrm{CCW}$ light. Before sending into the SOA, the $\mathrm{CCW}$ light is sent to an optical time delay (OTD) module to simulate the time delay $2 \Delta \mathrm{T}$ comparing with the $\mathrm{CW}$ light. The $\mathrm{CW}$ light should get the same time delay after the SOA propagation. So an OTD is set after the SOA in the CW light. Then the CW and $\mathrm{CCW}$ light are recombined and send into a photo detector to convert to electrical domain. An oscilloscope and a spectrometer are used to analyze the obtained signal.

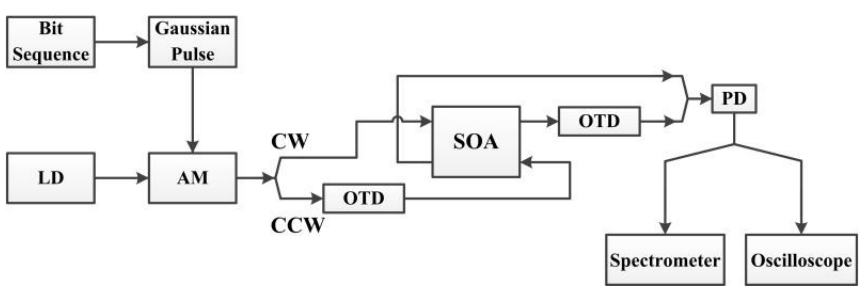

LD: Laser diode, AM: Amplitude modulator, PD: Photo detector OTD: Optical time delay module, SOA: Semiconductor optical amplifier

Fig. 3 Simulation experiment scheme based on the proposed method

The parameters of the simulation experiment is set as follows: the wavelength of the laser diode is $1552.52 \mathrm{~nm}$, the bit sequence is set as a fix pattern of one " 1 " per 16 bits at a repetition rate of $13.5 \mathrm{~Gb} / \mathrm{s}$, the bias current of the SOA is $618.2 \mathrm{~mA}$ and the time delay of the OTD is 70ps. UWB doublet pulse which is in very good shape and has a full width at half maximum (FWHM) of 56ps is obtained at the output of the PD, as shown in Fig. 4(a). The corresponding electrical spectrum of the generated doublet pulses is shown in Fig. 4(b). The central frequency is $6.05 \mathrm{GHz}$, and the $-10 \mathrm{~dB}$ bandwidth is $7.3 \mathrm{GHz}$ (from $2.4 \mathrm{GHz}$ to $9.7 \mathrm{GHz}$ ). It has a fractional bandwidth of $121 \%$.

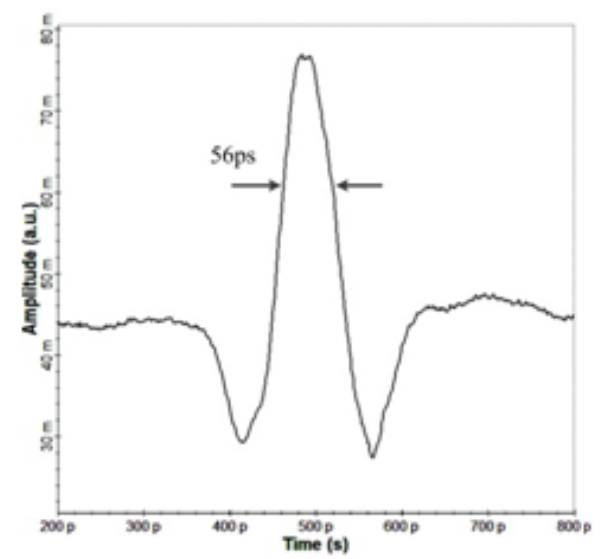

(a) 


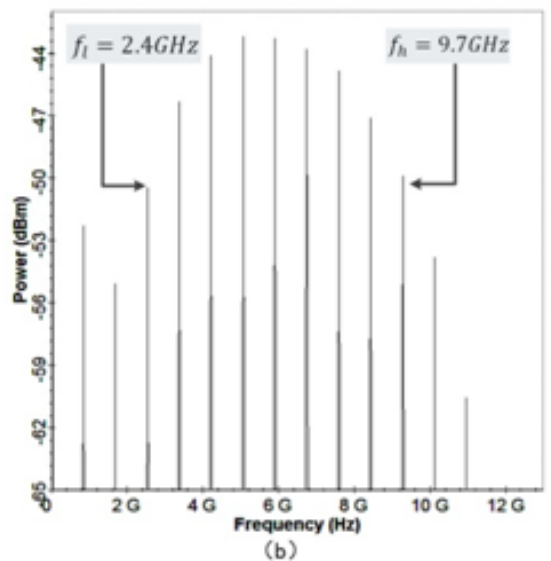

Fig. 4 Simulation experiment results, (a) generated UWB doublet pulse,(b) spectrum of generated UWB doublet pulses.

The FWHM of the generated UWB doublet pulses can be easily adjusted by tuning the time delay of the OTD [8]. So the central frequency and $-10 \mathrm{~dB}$ bandwidth of the UWB signal are variable. When the time delay of the OTD is changed from $30 \mathrm{ps}$ to $120 \mathrm{ps}$ and the other parameters remain the same, the central frequency and $-10 \mathrm{~dB}$ bandwidth of each UWB pulse is calculated, as shown in Fig. 5. The $-10 \mathrm{~dB}$ bandwidth becomes smaller and the central frequency shifts to the low frequency region simultaneously as the time delay of the OTD increases. Thus, UWB doublet signals with a tunable central frequency and $-10 \mathrm{~dB}$ bandwidth is obtained by adjusting the time delay of the OTD.

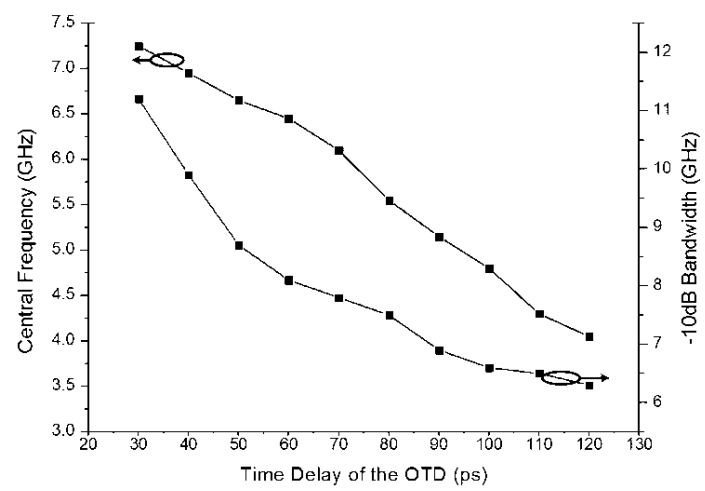

Fig. 5 Central frequency and $-10 \mathrm{~dB}$ bandwidth of the UWB doublet pulse under different time delay of the OTD

\section{IV . Conclusion}

In this paper, we have proposed a method to generate UWB doublet signals using a SOA based NOLM by exploiting the XPM effect. And the simulation experiment based on the proposed method has also been carried out. In the simulation experiment, the $\mathrm{CW}$ and $\mathrm{CCW}$ light are both phase modulated by each other in the SOA by the XPM effect, and the phase modulation is converted to intensity modulation in the NOLM output. UWB doublet pulses which meet the FCC regulations are generated. It has a central frequency of $6.05 \mathrm{GHz}$, the $-10 \mathrm{~dB}$ bandwidth is $7.3 \mathrm{GHz}$, and the fractional bandwidth is $121 \%$. By tuning the relative time delay between the CW light and the CCW light, UWB doublet signals with a tunable central frequency and $-10 \mathrm{~dB}$ bandwidth have also been generated.

\section{References}

[1] D. Porcine, P. Research, W. Hirt. "Ultra wideband radio technology: potential and challenges ahead". IEEE Commun Mag, 2003,41(7):66-74

[2] G.R. Aiello, G.D. Rogerson. "Ultra-wideband wireless systems". IEEE microwave magazine,2003,4(2):36-47.

[3] H. Nikookar, R. Prasad. "Introduction to Ultra Wideband for Wireless Commu nications". 2009:11-27.

[4] J.P. Yao, F. Zeng, Q. Wang, et al. "Photonic Generation of Ultrawideband Signals". Journal of Lightwave Technology, 2007, 25(11): 3219-3235

[5] J.P. Yao. "Photonics for ultrawideband communications". IEEE microwave magazine, 2009,10(4):82-95

[6] N.J. Doran, W. David. "Nonlinear-optical loop mirror". Optics Letters, 1988, 13(1): 56-58

[7] M.F. Faridus, M.H.A. Wahid, N. Sabani, et al. "SOA characterization for AND logic operation on SOA based NOLM". IEEE Regional Symposium on, 2011,9:372-376.

[8] B.W. Luo, J. Dong, Y. Yu et al. "Bandwidth-Tunable Single-Carrier UWB Monocycle Generation Using a Nonlinear Optical Loop Mirror”. IEEE Photonics Technology Letters,2012,24(18):1646-1649. 